\title{
The Efficacy of The Herbal Formula of Bunium Persicum and Coriandrum Sativum on Clinical Symptoms of Patients with Functional Dyspepsia: A Double-Blind Randomized Controlled Trial
}

Maryam Azimi

Kerman University of Medical Sciences

Mahboobeh Esfandiarpour

Kerman University of Medical Sciences

Mahboobeh Raeiszadeh

Kerman University of Medical Sciences

Ali Saeidpour Parizi ( $\triangle$ Ali45asp@yahoo.com )

Kerman University of Medical Sciences

\section{Research Article}

Keywords: Functional dyspepsia, Bunium persicum, Coriandrum sativum, Complementary and alternative medicine, Traditional medicine, Herbal medicine

Posted Date: February 18th, 2022

DOI: https://doi.org/10.21203/rs.3.rs-1182237/v1

License: (c) (i) This work is licensed under a Creative Commons Attribution 4.0 International License. Read Full License 


\section{Abstract}

\section{Background:}

Functional dyspepsia (FD) is one of the most prevalent chronic disorders affecting up to $30 \%$ of the world population with considerable impairment of quality of life. This study evaluated the efficacy of the herbal formula of Bunium persicum (Boiss.) B.Fedtsch. and Coriandrum Sativum L. on symptom severity, symptom frequency, and quality of life of patients with FD.

\section{Methods:}

This double-blind randomized controlled clinical trial, with parallel groups allocation ratio of 1:1, was conducted in a referral clinic of Afzalipour Hospital affiliated with Kerman University of Medical Sciences in Kerman, southeastern Iran.

A total of 90 patients with FD (diagnosed based on the Rome IV criteria) were included in this study. The patients with complications during the intervention, pregnancy or lactation, a history of allergy to herbal drugs, and severe organic or psychiatric disorders were excluded.

The participants received one capsule containing $500 \mathrm{mg}$ of herbal formula or placebo twice a day for 4 weeks and were subsequently followed up for 4 more weeks.

Clinical outcomes were assessed via the Dyspepsia Severity Questionnaire, the Rome IV Diagnostic Questionnaire, and Nepean Dyspepsia Index Questionnaire.

Results:

A total 40 participants in each group completed the follow-up period, and their data were analyzed statistically. All clinical outcomes showed significant improvements in herbal formula compared to the placebo without serious side effects $(P<0.05)$.

\section{Conclusions:}

Although this herbal formula can alleviate both epigastric pain syndrome (EPS) and postprandial distress syndrome (PDS), PDS seems to be improved higher than EPS.

\section{Trial registration:}

This trial was registered in the Iranian Registry of Clinical Trials (IRCT) under Reg. No. IRCT20190304042911N1 on 03/11/2019.

\section{Background}

Functional dyspepsia (FD) is one of the most prevalent chronic disorders with symptoms attributable to the upper or middle gastrointestinal tract, characterized by persistent and recurring symptoms such as postprandial fullness, early satiety, bloating, epigastric pain, epigastric burning, belching, nausea, and 
vomiting, which are not caused by structural or biochemical abnormalities. According to Rome IV criteria, FD is diagnosed with two, or more of the four main dyspepsia symptoms: postprandial fullness, early satiety, epigastric pain, and epigastric burning. Symptoms begin 6 months before diagnosis and typically last for 3 months (1). The pathophysiology of FD involves delayed gastric emptying, impaired post-meal gastric accommodation, hypersensitivity to gastric distention, altered duodenal sensitivity to lipids or acids, abnormal intestinal motility, genetic contributions, psychosocial factors, and central nervous system dysfunction $(2,3)$. Thus, FD is a common disorder affecting up to $30 \%$ of the world population with a considerable impact on emotional, physical, and psychological health, significantly impairing quality of life (4).

Acid-suppressive drugs, prokinetic agents, fundus-relaxing drugs, tricyclic antidepressants, and centrally active neuromodulators are currently recommended to treat FD. However, none of these therapies are effective in improving symptoms in a majority of patients without remarkable side effects (5-7).

The complementary and alternative medicine (CAM) has been widely used in treating functional gastrointestinal disorders (FGID) all over the world in recent years. The female gender, being white, high level of education, higher annual income, and comorbid medical conditions correlate directly with the use of CAM. The multi-component formula of herbal medicine, as the most frequently used modality of CAM in FGID, can modify GI function with a wide variety of mechanisms that have not been fully clarified $(8,9)$ in Asian countries for various medicinal purposes including gastrointestinal (diarrhea, flatulence) (10).

B. persicum is an important aromatic medical plant that belongs to the Apiaceae family with many bioactive components in its seeds including cuminaldehyde, $\alpha$-terpinene-7-al, $\gamma$-terpinene-7-al, $\gamma$-terpinene, $\rho$-cymene, $\beta$ Pinene, etc. Several pharmacological activities like antioxidant, anti-inflammatory, analgesic, acetylcholinesterase inhibitory, anticonvulsant, HMG-CoA reductase inhibitory, anticancer, anti-pancreatic lipase, antihistamine, antidiabetic, antispasmodic, antidiarrheal, antihepatotoxic, antityrosinase, and antimicrobial activities have been reported for this plant $(10,11)$. B. persicum can stimulate the intestinal motility and reduce significantly the time intervals to the passage of flatus, first bowel movement, and hospital discharge after a cesarean section $(12,13)$.

The hydroalcoholic extract of $B$. persicum was reported to be effective significantly in reducing the macroscopical and histopathological colon tissue injury in male Wistar rats (14). Moreover, adding $B$. persicum to the regular broiler chickens' diet causes a significant increase in the diameter of the mucosa, submucosa, muscularis, and serosa parts (15). Ethanol extract of $B$. persicum showed a significant dosedependent relaxing effect on dispersed intestinal smooth muscle cells of the guinea pig (16).

C. sativum belonging to Apiaceae is one of the most popularly used spices worldwide, with the main component of Linalool, and several biological activities such as antioxidant, anticancer, anxiolytic, anticonvulsant, analgesic, neuroprotective migraine-relieving, hypoglycemic, hypolipidemic, hypotensive, antimicrobial, and anti-inflammatory properties $(17,18)$.

It has been conducted that $C$. sativum seeds significantly improved the feed intake, surface area in absorptive villi segment, digestibility of proteins and fats, and growth performance in broiler chickens (19). Adding $C$. sativum to high fiber diets of Male albino rats significantly increased gastrointestinal motility (20). The 
protective and therapeutic effects of $C$. sativum in gastric ulcers have been reported in animal models. The protective effect of $C$. sativum has been found in indomethacin-induced ulcers $(21,22)$.

The present study aims to evaluate the efficacy and safety of the herbal formula of B. persicum and $C$. sativum on clinical symptoms of patients with functional dyspepsia and their quality of life (QOL).

\section{Methods}

\subsection{Study design}

This study is a double-blind randomized controlled clinical trial with parallel groups allocation ratio of 1:1.

\subsection{Study setting}

This study was conducted in a referral clinic of Afzalipour Hospital affiliated with Kerman University of Medical Sciences in Kerman, southeastern Iran. A trained gastroenterologist visited the patients. Next, the patients with the clinical diagnosis of functional dyspepsia were selected via a simple random sampling method. Finally, the patients who met the inclusion criteria were invited to the study.

\subsection{Participants}

Patients aged between 18 and 60 years, who developed functional dyspepsia based on the Rome IV, and met the inclusion criteria were entered in this study. In contrast, those with complications during the intervention or with a history of organic gastrointestinal disorders, pregnancy, or lactation, a history of allergy to herbal drugs, drug abuse, and severe organic or psychiatric disorders were excluded. The study was conducted in accordance with the declaration of Helsinki and subsequent revisions and approved by the ethical committee of Kerman University of Medical Sciences. Written informed consents were obtained before entering into the study.

\subsection{Randomization and blinding}

A biostatistician generated a randomization list via a blocked randomization method (non-stratified, four patients in each block) using Microsoft Excel software. All eligible patients were allocated sequentially by a trained secretory to the intervention and the control groups.

This study was a double-blind trial. The herbal formula was manufactured by a traditional pharmacist and was packaged and coded uniformly as a placebo. The person doing the coding had no role in the study until the end of the intervention. The patients were randomly assigned to one of the two study groups by a person who did not know how the drugs were coded. Statistical analysis of the data was performed by a third person who did not know how the groups were coded.

\subsection{Intervention}

\subsubsection{Plant material collection}

Dry fruits of $B$. persicum and $C$. sativum were purchased from the local herbal market, Kerman, Iran. The plants were authenticated at the Department of Traditional Pharmacy, Kerman University of Medical Sciences, 
Kerman; by Dr. Mahboobeh Raisezadeh. Besides, voucher specimens of B. persicum (KF-1141), and $C$. sativum (KF-1524) were deposited at the Herbarium of the Department of Pharmacognosy, Faculty of Pharmacy, Kerman University of Medical Sciences for future references.

\subsubsection{Plant material analysis}

To evaluate the quality of purchased plants, total ash, loss on drying, and extractive value percent were determined. Moreover, the total phenolic content of B. persicum and $C$. sativum ethanolic extract was evaluated spectrophotometrically using the Folin-Ciocalteu reagent assay according to a previously described method. Briefly, $100 \mu \mathrm{L}$ extract or a standard solution of gallic acid $(100-1000 \mu \mathrm{g} / \mathrm{ml})$ was mixed with $500 \mu \mathrm{L}$ of diluted Folin-Ciocalteu reagent (1:10 v/v) and was slightly shaken for a minute. Afterward, $400 \mu \mathrm{L}$ of an aqueous solution of $\mathrm{Na} 2 \mathrm{CO} 3(7.5 \% \mathrm{w} / \mathrm{v})$ was added and the obtained mixture was incubated for $30 \mathrm{~min}$ at room temperature in the dark. After incubation, $3000 \mu \mathrm{L}$ distilled water was added and the optical density was measured at $765 \mathrm{~nm}$ against a blank (ethanol 50\%) using a multi-mode microplate reader (BioTek ${ }^{8}$, USA). The total phenolic content of $B$. persicum and $C$. sativum extracts were expressed as $\mathrm{mg}$ of gallic acid equivalents (GAE)/g of the dry weight of extract (23).

\subsubsection{Formulation of herbal product}

Seeds of $B$. persicum, and $C$. sativum were equally soaked in twice the volume of grape vinegar purchased from Barij Essence Company, Iran for 24 hours, and then was dried in an oven at $40^{\circ} \mathrm{C}$. Afterward, the coarsely powdered seeds were packed in $500 \mathrm{mg}$ capsules after passing through mesh 30 . Cornstarch was also packed in similar capsules as a placebo.

\subsubsection{Group intervention:}

After obtaining written informed consent from the participants, they were randomly divided into the intervention and control groups. The patients were advised to take one capsule of the herbal formula in the intervention group and one capsule of placebo in the control group, with a glass of water, two times a day, after meals, for 4 weeks, and to subsequently follow it up for another 4 weeks.

\subsection{Primary outcomes}

\subsubsection{Dyspepsia Symptoms Severity}

Dyspepsia Symptoms Severity were evaluated through the standard eight-item questionnaire (4-Likert scale: 1-4) designed to assess the presence and severity of dyspepsia, with the following 8 items: epigastric pain, epigastric burning, bloating, early satiety, belching, postprandial fullness, nausea, and vomiting. The minimum and maximum scores obtained from this questionnaire are 8 and 32 (24).

\subsubsection{Dyspepsia Symptoms Frequency}

The Dyspepsia Symptoms were assessed through the standard eight-item questionnaire (6-Likert scale: 1-6) designed by Rome IV foundation to assess the presence and frequency of dyspepsia, with the following 8 items: epigastric pain, epigastric burning, bloating, early satiety, belching, postprandial fullness, nausea, and vomiting. The minimum and maximum scores obtained from this questionnaire are 8 and 48 (25). 


\subsubsection{Dyspepsia Quality of life}

The quality of life of participants was assessed via the 10-item Nepean Dyspepsia Index (NDI-10). This questionnaire contains 10 questions (5-Likert scale: 1 -5) organized into the following 5 subscales: tension, interference with daily activities, eating/drinking, knowledge/control, and work/study. The minimum and maximum scores obtained from this questionnaire are 10 and $50(26,27)$.

\subsection{Secondary outcome}

\subsubsection{Drug adverse effects}

Drug adverse effects such as rash, diarrhea, abdominal pain, nausea, and bloating were assessed during the study through a patient-reported adverse effect questionnaire.

\subsection{Sample size}

According to the parameters related to the QOL of the intervention group (baseline and after 4 weeks) obtained from the pilot study and regarding the Type I and Type II errors of 0.05 and 0.2 , the effect size of 3 , and a dropout rate of $10 \%$; the final sample size of 40 was calculated for each group via the following formula:

$$
n=\frac{\left(Z_{1-1 e x \$ \alpha \$ /-1 e x \$ 2 \$}+Z_{1-\beta}\right)^{2} *\left({\sigma_{1}}^{2}+\sigma_{2}^{2}\right)}{d^{2}}
$$

$z_{0.975}=1.96 ; z_{0.8}=0.84 ; \alpha=0.05 ; \beta=0.2 ; \sigma_{1}=5.74 ; \sigma_{2}=3.25 ; \mathrm{d}=3$

\subsection{Statistical analysis}

The participants' demographic data were analyzed using descriptive statistics (mean, standard deviation, skewness, and kurtosis). Moreover, to compare the changes in the symptoms severity, symptoms frequency, and quality of life between the two groups at three different time points (baseline, 4, and 8 weeks), the independent sample t-test, the paired sample t-test, the chi-square test, one-way analysis of covariance (oneway ANOVA), and the multivariate analysis of covariance (MANOVA) were conducted. The statistical analysis was carried out using SPSS 26 at the significance level of $0.05(p<0.05)$.

\section{Results}

\subsection{Phytochemical analysis of herbal formula}

Total ash of B. persicum and C. sativum were determined as $6.8 \%$, and $4.8 \%$. Total phenolic content (Gallic acid equivalent $/ \mathrm{mg} / \mathrm{g}$ ) of hydroalcoholic extracts of B. persicum, and $C$. sativum were calculated to be 204.5 \pm 8.9 , and $182.7 \pm 11.4$ (Table 1 ). 
Table 1

Results of the analysis of B. persicum and C. sativum fruits

\begin{tabular}{|lllll|}
\hline $\begin{array}{l}\text { Plant } \\
\text { material }\end{array}$ & $\begin{array}{l}\text { Total ash } \\
(\%)\end{array}$ & $\begin{array}{l}\text { Loss on drying } \\
(\%)\end{array}$ & $\begin{array}{l}\text { Extract yield } \\
\text { (\%W/W) }\end{array}$ & $\begin{array}{l}\text { Total phenolic (mg gallic acid/ g } \\
\text { extract) }\end{array}$ \\
\hline B. persicum & $6.8 \pm 0.3$ & $5.1 \pm 0.4$ & $21.7 \pm 1.1$ & $204.5 \pm 8.9$ \\
\hline C. sativum & $4.8 \pm 0.1$ & $3.7 \pm 0.3$ & $23.1 \pm 1.2$ & $182.7 \pm 11.4$ \\
\hline
\end{tabular}

\subsection{Flowchart of the study}

In total, 90 elderly patients with constipation were randomly assigned to either herbal formula or placebo groups. The participants were selected from the patients who completed screening tests for two weeks, met the inclusion criteria, and signed the informed consent form. In both groups, 5 patients withdrew before completing the assessment due to irregular drug intake, or arbitrary consumption of other related drugs. Finally, 40 participants in each group completed the follow-up period and their data were analyzed statistically (Fig. 1).

\subsection{Demographic characteristics}

The mean age of the patients in the intervention group was 38.08 in the range of 18 to 60 years, and in the control group was 37.32 in the range of 18 to 56 years. Moreover, $67.5 \%$ of the patients in the intervention group and $70 \%$ of them in the control group were female. Therefore, there were no significant differences between the two groups in terms of gender and age $(p>0.05)$ (Table 2$)$.

Table 2

Comparison of the demographic variables among two groups

\begin{tabular}{|c|c|c|c|c|}
\hline \multicolumn{2}{|l|}{ Demographic variables } & \multicolumn{2}{|l|}{ Groups } & \multirow[t]{3}{*}{$P$ value } \\
\hline & & Intervention & Control & \\
\hline & & $(n=40)$ & $(n=40)$ & \\
\hline \multirow[t]{2}{*}{ Sex: Number $(\%) \Rightarrow$} & Man & $13(32.5 \%)$ & $12(30 \%)$ & \multirow[t]{2}{*}{0.809} \\
\hline & Woman & $27(67.5 \%)$ & $28(70 \%)$ & \\
\hline \multicolumn{2}{|l|}{ Age: Mean (SD) $\Rightarrow \Rightarrow$} & $38.07(10.40)$ & $37.32(10.34)$ & 0.747 \\
\hline
\end{tabular}

\subsection{Quality of life}

Quality of life datasets typically followed a normal distribution, but there were no linear relationships among the two pairs of dependent variables $(F=115.112, \mathrm{P}<0.001)$. So, we used the independent samples t-test to compare the QOL between the groups. Levine's test indicated the homogeneity of variances $(F=0.212, P=$ 0.646). The mean differential score of the QOL was lower in the intervention group than the control group that 
showed the significant improvement of QOL with using of the herbal formula compared to the placebo $(\mathrm{P}<$ $0.001)$ that was remained until the end of the follow-up period $(P=0.091)$. Mean difference of -7.30 , and Hedge's effect size of 1.681 showed a significant difference between the two groups (Tables $3-5$ ).

Table 3

Comparison of the QOL, and total symptom severity

\begin{tabular}{|c|c|c|c|c|c|c|c|}
\hline \multirow[t]{2}{*}{ Variables } & \multirow[t]{2}{*}{$\begin{array}{l}\text { Independent } \\
\text { t-test }\end{array}$} & \multirow[t]{2}{*}{$\begin{array}{l}\text { Degree of } \\
\text { freedom }\end{array}$} & \multirow[t]{2}{*}{$\begin{array}{l}P \\
\text { value }\end{array}$} & \multirow[t]{2}{*}{$\begin{array}{l}\text { Mean } \\
\text { difference }\end{array}$} & \multirow[t]{2}{*}{$\begin{array}{l}\text { Effect size } \\
\text { Hedge 's g }\end{array}$} & \multicolumn{2}{|c|}{$\begin{array}{l}95 \% \text { confidence } \\
\text { interval }\end{array}$} \\
\hline & & & & & & lower & Upper \\
\hline $\begin{array}{l}\text { Quality of } \\
\text { life }\end{array}$ & -7.594 & 78 & $<.001$ & -7.30 & 1.681 & -9.213 & -5.386 \\
\hline $\begin{array}{l}\text { Symptoms } \\
\text { severity }\end{array}$ & -8.229 & 78 & $<.001$ & -5.975 & 1.824 & -7.420 & -4.529 \\
\hline
\end{tabular}

\subsection{Symptoms severity}

\subsubsection{Total severity of FD}

All data related to total symptom severity had a normal distribution, but no linear relationship $(F=79.263, P<$ $0.001)$ and no significant value were found in Levine's test $(F=2.479, P=0.119)$. According to the independent samples t-test, the mean differential score of total symptoms severity was lower in the intervention group compared to the control group $(P<0.001)$. The mean difference of -5.974 , and Hedge's effect size of 1.824 showed a significant difference between the two groups (Tables $3-5$ ).

\subsubsection{Severity of symptoms:}

We evaluated all data relating to the severity of eight symptoms (postprandial fullness, early satiety, bloating, belching, nausea, vomiting, epigastric pain, and epigastric burning) in the two groups as follows: The acceptance rate of skewness and kurtosis $( \pm 2, \pm 3)$ confirmed the normal distribution of all data. However, the homogeneity of regression (Wilk's Lambda, $\mathrm{P}<0.001$ ), and the homogeneity of variance and covariance (Box's $M=125.495, P<0.001)$ were not established. Moreover, the homogeneity of variance was confirmed except for postprandial fullness, belching, and vomiting (Leven's test, $P>0.001$ ).

According to the $\mathrm{F}$ test, all symptoms were improved significantly in the intervention group compared to the control group $(P>0.05)$. Furthermore, evaluating the effect size showed that herbal formula was more effective in improving bloating, epigastric pain, postprandial fullness, and early satiety. But this declining trend did not continue to the end of the follow-up period in the 8th week (except for epigastric pain and vomiting) (Tables 4-7). 
Table 4

Descriptive indicators of research variables

\begin{tabular}{|c|c|c|c|c|c|}
\hline \multirow[t]{2}{*}{ Variable } & \multirow[t]{2}{*}{ Group } & \multirow{2}{*}{$\begin{array}{l}\text { Descriptive } \\
\text { indicators }\end{array}$} & \multicolumn{3}{|l|}{ Time } \\
\hline & & & Baseline & $\begin{array}{l}\text { After } 4 \\
\text { weeks }\end{array}$ & After 8 weeks \\
\hline \multirow[t]{4}{*}{ Quality of life } & \multirow[t]{2}{*}{ Intervention } & Mean \pm SD & $5.74 \pm 28.65$ & $3.25 \pm 17.40$ & $2.85 \pm 18.02$ \\
\hline & & Skewness-Kurtosis & $0.092-0.201$ & $\begin{array}{l}0.532- \\
0.708\end{array}$ & $0.148-0.451$ \\
\hline & \multirow[t]{2}{*}{ Control } & Mean \pm SD & $6.58 \pm 29.37$ & $5.34 \pm 25.42$ & $5.32 \pm 25.55$ \\
\hline & & Skewness-Kurtosis & $\begin{array}{l}-0.210- \\
-0.380\end{array}$ & $\begin{array}{l}0.273- \\
2.195\end{array}$ & $0.283-1.462$ \\
\hline \multirow{4}{*}{$\begin{array}{l}\text { Symptoms } \\
\text { severity }\end{array}$} & \multirow[t]{2}{*}{ Intervention } & Mean \pm SD & $4.49 \pm 19.65$ & $2.26 \pm 10.95$ & $3.08 \pm 12.50$ \\
\hline & & Skewness-Kurtosis & $\begin{array}{l}-0.046-- \\
1.245\end{array}$ & $\begin{array}{l}0.177-- \\
0.927\end{array}$ & $\begin{array}{l}-0.046-- \\
1.245\end{array}$ \\
\hline & \multirow[t]{2}{*}{ Control } & Mean \pm SD & $3.68 \pm 18.47$ & $3.64 \pm 15.75$ & $4.08 \pm 16.95$ \\
\hline & & Skewness-Kurtosis & $\begin{array}{l}0.102- \\
-0.303\end{array}$ & $\begin{array}{l}0.151- \\
0.578\end{array}$ & $-0 / 090-0.145$ \\
\hline \multirow{4}{*}{$\begin{array}{l}\text { Postprandial } \\
\text { fullness }\end{array}$} & \multirow[t]{2}{*}{ Intervention } & Mean \pm SD & $1.06 \pm 2.70$ & $0.47 \pm 1.32$ & $0.50 \pm 1.55$ \\
\hline & & Skewness-Kurtosis & $-0.421--1.01$ & $0.77--1.47$ & $-0.209--2.06$ \\
\hline & \multirow[t]{2}{*}{ Control } & Mean \pm SD & $1.03 \pm 2.50$ & $0.89 \pm 2.15$ & $1.03 \pm 2.40$ \\
\hline & & Skewness-Kurtosis & $-0.290--1.11$ & $\begin{array}{l}0.147- \\
-0.919\end{array}$ & $-0.159--1.21$ \\
\hline \multirow[t]{4}{*}{ Bloating } & \multirow[t]{2}{*}{ Intervention } & Mean \pm SD & $1.01 \pm 3.01$ & $0.48 \pm 1.35$ & $0.48 \pm 1.65$ \\
\hline & & Skewness-Kurtosis & $-0.780-0.402$ & $0.654--1.65$ & $-0.654--1.65$ \\
\hline & \multirow[t]{2}{*}{ Control } & Mean \pm SD & $1.09 \pm 2.80$ & $1.06 \pm 2.52$ & $1.12 \pm 2.65$ \\
\hline & & Skewness-Kurtosis & $-0.329--1.21$ & $-0.136--1.17$ & $-0.281--1.27$ \\
\hline \multirow[t]{4}{*}{ Early satiety } & \multirow[t]{2}{*}{ Intervention } & Mean \pm SD & $0.96 \pm 2.27$ & $0.38 \pm 1.17$ & $0.50 \pm 1.42$ \\
\hline & & Skewness-Kurtosis & $-0.046--1.13$ & $1.77-1.22$ & $0.315--2.01$ \\
\hline & \multirow[t]{2}{*}{ Control } & Mean \pm SD & $0.99 \pm 1.97$ & $0.83 \pm 1.75$ & $0.91 \pm 1.87$ \\
\hline & & Skewness-Kurtosis & $0.376--1.32$ & $\begin{array}{l}0.786-- \\
0.311\end{array}$ & $0.472--1.13$ \\
\hline \multirow[t]{4}{*}{ Belching } & \multirow[t]{2}{*}{ Intervention } & Mean \pm SD & $1.15 \pm 2.10$ & $0.43 \pm 1.25$ & $0.78 \pm 1.50$ \\
\hline & & Skewness-Kurtosis & $0.434--1.34$ & $1.20--0.592$ & $1.17--0.284$ \\
\hline & \multirow[t]{2}{*}{ Control } & Mean \pm SD & $0.99 \pm 1.87$ & $0.82 \pm 1.67$ & $0.96 \pm 1.80$ \\
\hline & & Skewness-Kurtosis & $0.594--1.07$ & $0.972-$ & $0.783--0 / 700$ \\
\hline
\end{tabular}




\begin{tabular}{|c|c|c|c|c|c|}
\hline & & & & 0.067 & \\
\hline \multirow[t]{4}{*}{ Nausea } & \multirow[t]{2}{*}{ Intervention } & Mean \pm SD & $1.20 \pm 2.02$ & $0.62 \pm 1.37$ & $0.71 \pm 1.47$ \\
\hline & & Skewness-Kurtosis & $0.684--1.16$ & $1.48-1.15$ & $1.19-0.067$ \\
\hline & \multirow[t]{2}{*}{ Control } & Mean \pm SD & $1.08 \pm 1.90$ & $0.71 \pm 1.55$ & $0.81 \pm 1.65$ \\
\hline & & Skewness-Kurtosis & $0.592--1.26$ & $\begin{array}{l}0.926- \\
-0.408\end{array}$ & $1.01--0.130$ \\
\hline \multirow[t]{4}{*}{ Vomiting } & \multirow[t]{2}{*}{ Intervention } & Mean \pm SD & $0.84 \pm 1.50$ & $0.30 \pm 1.10$ & $0.36 \pm 1.15$ \\
\hline & & Skewness-Kurtosis & $1.73-2.29$ & $2.77-2.97$ & $2.03-2.26$ \\
\hline & \multirow[t]{2}{*}{ Control } & Mean \pm SD & $0.67 \pm 1.40$ & $0.50 \pm 1.27$ & $0.56 \pm 1.30$ \\
\hline & & Skewness-Kurtosis & $1.45-0.862$ & $1.65-2.01$ & $1.77-2.33$ \\
\hline \multirow[t]{4}{*}{ Epigastric pain } & \multirow[t]{2}{*}{ Intervention } & Mean \pm SD & $0.84 \pm 3.17$ & $0.65 \pm 1.67$ & $0.68 \pm 1.80$ \\
\hline & & Skewness-Kurtosis & $-0.889-0.394$ & $1.02-2.55$ & $0.780-1.45$ \\
\hline & \multirow[t]{2}{*}{ Control } & Mean \pm SD & $0.98 \pm 2.95$ & $0.90 \pm 2.42$ & $0.95 \pm 2.57$ \\
\hline & & Skewness-Kurtosis & $\begin{array}{l}-0.576- \\
-0.668\end{array}$ & $\begin{array}{l}0.125- \\
-0.657\end{array}$ & $\begin{array}{l}-0.133- \\
-0.843\end{array}$ \\
\hline \multirow[t]{4}{*}{ Epigastric burning } & \multirow[t]{2}{*}{ Intervention } & Mean \pm SD & $0.88 \pm 2.87$ & $0.68 \pm 1.70$ & $0.71 \pm 1.95$ \\
\hline & & Skewness-Kurtosis & $\begin{array}{l}0.924- \\
0.520\end{array}$ & $\begin{array}{c}0.470- \\
-0.759\end{array}$ & $0.073--0.959$ \\
\hline & \multirow[t]{2}{*}{ Control } & Mean \pm SD & $0.79 \pm 3.07$ & $0.90 \pm 2.40$ & $0.91 \pm 2.70$ \\
\hline & & Skewness-Kurtosis & $\begin{array}{l}-0.419- \\
-0.469\end{array}$ & $\begin{array}{l}-0.018- \\
-0.721\end{array}$ & $0.778-0.649$ \\
\hline
\end{tabular}

Table 5

Comparison of the QOL, and total symptom severity

\begin{tabular}{|c|c|c|c|c|c|c|c|}
\hline \multirow[t]{2}{*}{ Variables } & \multirow[t]{2}{*}{$\begin{array}{l}\text { Independent } \\
\text { t-test }\end{array}$} & \multirow[t]{2}{*}{$\begin{array}{l}\text { Degree of } \\
\text { freedom }\end{array}$} & \multirow[t]{2}{*}{$\begin{array}{l}P \\
\text { value }\end{array}$} & \multirow[t]{2}{*}{$\begin{array}{l}\text { Mean } \\
\text { difference }\end{array}$} & \multirow[t]{2}{*}{$\begin{array}{l}\text { Effect size } \\
\text { Hedge's g }\end{array}$} & \multicolumn{2}{|c|}{$\begin{array}{l}95 \% \text { confidence } \\
\text { interval }\end{array}$} \\
\hline & & & & & & lower & Upper \\
\hline $\begin{array}{l}\text { Quality of } \\
\text { life }\end{array}$ & -7.594 & 78 & $<.001$ & -7.30 & 1.681 & -9.213 & -5.386 \\
\hline $\begin{array}{l}\text { Symptoms } \\
\text { severity }\end{array}$ & -8.229 & 78 & $<.001$ & -5.975 & 1.824 & -7.420 & -4.529 \\
\hline
\end{tabular}


Table 6

Comparison the quality of life, and the severity of symptoms

\begin{tabular}{|c|c|c|c|c|c|c|c|c|}
\hline \multirow[t]{2}{*}{ Variables } & \multicolumn{4}{|c|}{ Baseline-After 4 weeks } & \multicolumn{4}{|c|}{ After 4 weeks-After 8 weeks } \\
\hline & $\begin{array}{l}\text { Mean } \\
\text { deviation }\end{array}$ & $\mathbf{T}$ & $\begin{array}{l}\text { Degree of } \\
\text { freedom }\end{array}$ & $\begin{array}{l}P \\
\text { value }\end{array}$ & $\begin{array}{l}\text { Mean } \\
\text { deviation }\end{array}$ & $\mathbf{T}$ & $\begin{array}{l}\text { Degree } \\
\text { of } \\
\text { freedom }\end{array}$ & $\begin{array}{l}P \\
\text { value }\end{array}$ \\
\hline Quality of life & 11.250 & 16.849 & 39 & $<0.001$ & -0.625 & -1.731 & 39 & 0.091 \\
\hline $\begin{array}{l}\text { Total } \\
\text { symptoms } \\
\text { severity }\end{array}$ & 8.700 & 14.809 & 39 & $\begin{array}{l}< \\
0.001\end{array}$ & -1.550 & -3.969 & 39 & $\begin{array}{l}< \\
0.001\end{array}$ \\
\hline $\begin{array}{l}\text { Postprandial } \\
\text { fullness }\end{array}$ & 1.375 & 8.883 & 39 & $<0.001$ & -0.225 & -3.365 & 39 & 0.002 \\
\hline Early satiety & 1.100 & 7.986 & 39 & $<001$ & -0.250 & -3.606 & 39 & $\begin{array}{l}< \\
0.001\end{array}$ \\
\hline Bloating & 1.650 & 12.519 & 39 & $<001$ & -0.300 & -4.088 & 39 & $\begin{array}{l}< \\
0.001\end{array}$ \\
\hline Belching & 0.850 & 6.224 & 39 & $<001$ & -0.250 & -2.912 & 39 & 0.006 \\
\hline Nausea & 0.650 & 4.932 & 39 & $<.001$ & -0.100 & -2.082 & 39 & 0.044 \\
\hline Vomiting & 0.400 & 1.122 & 39 & 0.003 & -0.500 & -1.433 & 39 & 0.160 \\
\hline Epigastric pain & 1.500 & 12.093 & 39 & $<_{0.001}$ & -0.125 & -1.302 & 39 & 0.200 \\
\hline $\begin{array}{l}\text { Epigastric } \\
\text { burning }\end{array}$ & 1.175 & 10.436 & 39 & $<.001$ & -0.250 & -2.912 & 39 & 0.006 \\
\hline
\end{tabular}


Table 7

results of one-way analysis of variance based on differential score related to intergroup difference in severity of symptoms

\begin{tabular}{|c|c|c|c|c|c|c|c|c|}
\hline Variables & MD & $\begin{array}{l}\text { SS } \\
\text { (experimental) }\end{array}$ & $\begin{array}{l}\text { SS } \\
\text { (error) }\end{array}$ & $\begin{array}{l}\text { MS } \\
\text { (experiment) }\end{array}$ & $\begin{array}{l}\text { MS } \\
\text { (error) }\end{array}$ & $F$ & $\mathrm{P}^{*}$ & $\bigotimes^{2}$ ** \\
\hline Fullness & 1.025 & 21.012 & 46.475 & 21.012 & 0.596 & 35.266 & $\stackrel{<}{<.001}$ & 0.311 \\
\hline Satiety & 0.875 & 15.313 & 38.575 & 15.313 & 0.495 & 30.962 & $<.001$ & 0.284 \\
\hline Bloating & 1.375 & 37.813 & 34.075 & 37.813 & 0.475 & 79.552 & $<.001$ & 0.505 \\
\hline Belching & 0.650 & 8.450 & 39.500 & 8.450 & 0.506 & 16.686 & $\stackrel{<}{<.001}$ & 0.176 \\
\hline Nausea & 0.300 & 1.800 & 36.200 & 1.800 & 0.462 & 3.878 & 0.052 & 0.047 \\
\hline Vomiting & 0.275 & 1.513 & 29.975 & 1.513 & 0.384 & 3.936 & 0.051 & 0.048 \\
\hline Pain & 0.975 & 19.02 & 41.975 & 19.02 & 0.538 & 35.330 & $\begin{array}{l}< \\
0.001\end{array}$ & 0.312 \\
\hline Burning & 0.500 & 5 & 38.550 & 5 & 0.494 & 10.117 & 0.002 & 0.115 \\
\hline
\end{tabular}

\subsection{Frequency of symptoms}

There was no significant difference between the two groups in the term of symptoms frequency at baseline ( $P$ $>0.05$ ). The symptoms frequency of the patients significantly reduced after 4 weeks in all dimensions in the herbal formula group compared to the placebo; and remained significant to the end of the 8th week $(P<0.05)$. At the beginning of the study, the daily prevalence of symptoms in the herbal formula group was as follows: postprandial fullness (40\%), epigastric pain (37.5\%), epigastric burning (25\%), belching ( $25 \%)$, bloating (22.5\%), nausea (12.5\%), early satiety (10\%), and vomiting (5\%). After 4 weeks, and until the end of 8 weeks; none of the symptoms was reported daily (Table 8 ). 
Table 8

Comparison the frequency of symptoms

\begin{tabular}{|c|c|c|c|c|c|c|c|}
\hline \multirow[t]{2}{*}{ Variables } & \multirow[t]{2}{*}{ Frequency } & \multicolumn{2}{|l|}{ Baseline } & \multicolumn{2}{|l|}{ After 4 weeks } & \multicolumn{2}{|c|}{ After 8 weeks } \\
\hline & & Intervention & Control & Intervention & Control & Intervention & Control \\
\hline \multirow[t]{7}{*}{$\begin{array}{l}\text { Postprandial } \\
\text { fullness }\end{array}$} & $\begin{array}{l}<1 \\
\text { day/month }\end{array}$ & $8(20 \%)$ & $4(10 \%)$ & $30(75 \%)$ & $\begin{array}{l}7 \\
(17.5 \%)\end{array}$ & 17 (42.5\%) & $6(15 \%)$ \\
\hline & $\begin{array}{l}1 \text { day/ } \\
\text { month }\end{array}$ & $6(15 \%)$ & $\begin{array}{l}9 \\
(22.5 \%)\end{array}$ & 9 (22.5\%) & $\begin{array}{l}12 \\
(30 \%)\end{array}$ & 17 (42.5\%) & $\begin{array}{l}10 \\
(25 \%)\end{array}$ \\
\hline & $\begin{array}{l}2- \\
3 / \text { month }\end{array}$ & $1(2.5 \%)$ & $\begin{array}{l}16 \\
(15 \%)\end{array}$ & $0(0 \%)$ & $\begin{array}{l}5 \\
(12.5 \%)\end{array}$ & $5(12.5 \%)$ & $\begin{array}{l}5 \\
(12.5 \%)\end{array}$ \\
\hline & $\begin{array}{l}1 \\
\text { day/week }\end{array}$ & $2(5 \%)$ & $4(10 \%)$ & $1(2,5 \%)$ & $8(20 \%)$ & $1(2.5 \%)$ & $8(20 \%)$ \\
\hline & $\begin{array}{l}>1 \\
\text { day/week }\end{array}$ & 7 (17.5\%) & $\begin{array}{l}10 \\
(25 \%)\end{array}$ & $0(0 \%)$ & $8(20 \%)$ & $0(0 \%)$ & $\begin{array}{l}10 \\
(25 \%)\end{array}$ \\
\hline & Daily & $16(40 \%)$ & $\begin{array}{l}7 \\
(17.5 \%)\end{array}$ & $0(0 \%)$ & $0(0 \%)$ & $0(0 \%)$ & $\begin{array}{l}1 \\
(2.5 \%)\end{array}$ \\
\hline & & 0.069 & & $<0.001$ & & $<0.001$ & \\
\hline \multirow[t]{7}{*}{ Early satiety } & $\begin{array}{l}<1 \\
\text { day/month }\end{array}$ & $12(30 \%)$ & $\begin{array}{l}16 \\
(40 \%)\end{array}$ & $28(70 \%)$ & $\begin{array}{l}17 \\
(42.5 \%)\end{array}$ & 22 (55\%) & $\begin{array}{l}17 \\
(42.5 \%)\end{array}$ \\
\hline & $\begin{array}{l}1 \text { day/ } \\
\text { month }\end{array}$ & $7(17.5 \%)$ & $\begin{array}{l}7 \\
(17.5 \%)\end{array}$ & $10(25 \%)$ & $\begin{array}{l}9 \\
(22.5 \%)\end{array}$ & $16(40 \%)$ & $8(20 \%)$ \\
\hline & $\begin{array}{l}2- \\
3 / \text { month }\end{array}$ & $6(15 \%)$ & $\begin{array}{l}5 \\
(12.5 \%)\end{array}$ & $2(5 \%)$ & $6(15 \%)$ & $2(5 \%)$ & $\begin{array}{l}5 \\
(12.5 \%)\end{array}$ \\
\hline & $\begin{array}{l}1 \\
\text { day/week }\end{array}$ & $0(0 \%)$ & $\begin{array}{l}3 \\
(7.5 \%)\end{array}$ & $0(0 \%)$ & $6(15 \%)$ & $0(0 \%)$ & $8(20 \%)$ \\
\hline & $\begin{array}{l}>1 \\
\text { day/week }\end{array}$ & 11 (27.5\%) & $8(20 \%)$ & $0(0 \%)$ & $2(5 \%)$ & $0(0 \%)$ & $2(5 \%)$ \\
\hline & Daily & $4(10 \%)$ & $\begin{array}{l}1 \\
(2.5 \%)\end{array}$ & $0(0 \%)$ & $0(0 \%)$ & $0(0 \%)$ & $0(0 \%)$ \\
\hline & & 0.312 & & 0.013 & & 0.006 & \\
\hline \multirow[t]{5}{*}{ Bloating } & $\begin{array}{l}<1 \\
\text { day/month }\end{array}$ & $5(12.5 \%)$ & $6(15 \%)$ & $26(65 \%)$ & $\begin{array}{l}10 \\
(25 \%)\end{array}$ & $8(20 \%)$ & $\begin{array}{l}16 \\
(40 \%)\end{array}$ \\
\hline & $\begin{array}{l}1 \text { day/ } \\
\text { month }\end{array}$ & $4(10 \%)$ & $6(15 \%)$ & $12(30 \%)$ & $\begin{array}{l}7 \\
(17.5 \%)\end{array}$ & $7(17.5 \%)$ & $\begin{array}{l}18 \\
(45 \%)\end{array}$ \\
\hline & $\begin{array}{l}2- \\
3 / \text { month }\end{array}$ & $2(5 \%)$ & $\begin{array}{l}5 \\
(12.5 \%)\end{array}$ & $2(5 \%)$ & $\begin{array}{l}3 \\
(7.5 \%)\end{array}$ & $5(12.5 \%)$ & $4(10 \%)$ \\
\hline & $\begin{array}{l}1 \\
\text { day/week }\end{array}$ & $6(15 \%)$ & $4(10 \%)$ & $0(0 \%)$ & $\begin{array}{l}7 \\
(17.5 \%)\end{array}$ & $4(10 \%)$ & $\begin{array}{l}1 \\
(2.5 \%)\end{array}$ \\
\hline & $\begin{array}{l}>1 \\
\text { day/week }\end{array}$ & $14(35 \%)$ & $8(20 \%)$ & $0(0 \%)$ & $8(20 \%)$ & $0(0 \%)$ & $\begin{array}{l}11 \\
(27.5 \%)\end{array}$ \\
\hline
\end{tabular}




\begin{tabular}{|c|c|c|c|c|c|c|c|}
\hline \multirow[t]{2}{*}{ Variables } & \multirow[t]{2}{*}{ Frequency } & \multicolumn{2}{|l|}{ Baseline } & \multicolumn{2}{|c|}{ After 4 weeks } & \multicolumn{2}{|c|}{ After 8 weeks } \\
\hline & & Intervention & Control & Intervention & Control & Intervention & Control \\
\hline & Daily & $11(27.5 \%)$ & $\begin{array}{l}9 \\
(22.5 \%)\end{array}$ & $0(0 \%)$ & $\begin{array}{l}5 \\
(12.5 \%)\end{array}$ & $0(0 \%)$ & $6(15 \%)$ \\
\hline & & 0.548 & & $<0.001$ & & $<0.001$ & \\
\hline \multirow[t]{7}{*}{ Belching } & $\begin{array}{l}<1 \\
\text { day/month }\end{array}$ & $16(40 \%)$ & $\begin{array}{l}18 \\
(45 \%)\end{array}$ & 27 (67.5\%) & $\begin{array}{l}21 \\
(52.5 \%)\end{array}$ & $25(62.5 \%)$ & $\begin{array}{l}21 \\
(52.5 \%)\end{array}$ \\
\hline & $\begin{array}{l}1 \text { day/ } \\
\text { month }\end{array}$ & 7 (17.5\%) & $4(10 \%)$ & $4(10 \%)$ & $4(10 \%)$ & $5(12.5 \%)$ & $\begin{array}{l}3 \\
(7.5 \%)\end{array}$ \\
\hline & $\begin{array}{l}2- \\
3 / \text { month }\end{array}$ & $2(5 \%)$ & $4(10 \%)$ & 9 (22.5\%) & $\begin{array}{l}5 \\
(12.5 \%)\end{array}$ & $8(20 \%)$ & $\begin{array}{l}3 \\
(7.5 \%)\end{array}$ \\
\hline & $\begin{array}{l}1 \\
\text { day/week }\end{array}$ & $2(5 \%)$ & $\begin{array}{l}5 \\
(12.5 \%)\end{array}$ & $0(0 \%)$ & $\begin{array}{l}5 \\
(12.5 \%)\end{array}$ & $2(5 \%)$ & $\begin{array}{l}7 \\
(17.5 \%)\end{array}$ \\
\hline & $\begin{array}{l}>1 \\
\text { day/week }\end{array}$ & $3(7.5 \%)$ & $\begin{array}{l}7 \\
(17.5 \%)\end{array}$ & $0(0 \%)$ & $4(10 \%)$ & $0(0 \%)$ & $4(10 \%)$ \\
\hline & Daily & $10(25 \%)$ & $2(5 \%)$ & $0(0 \%)$ & $\begin{array}{l}1 \\
(2.5 \%)\end{array}$ & $0(0 \%)$ & $2(5 \%)$ \\
\hline & & 0.08 & & 0.036 & & 0.036 & \\
\hline \multirow[t]{6}{*}{ Nausea } & $\begin{array}{l}<1 \\
\text { day/month }\end{array}$ & $20(50 \%)$ & $\begin{array}{l}21 \\
(52.5 \%)\end{array}$ & 27 (67.5\%) & $\begin{array}{l}25 \\
(62.5 \%)\end{array}$ & 25 (62.5\%) & $\begin{array}{l}23 \\
(57.5 \%)\end{array}$ \\
\hline & $\begin{array}{l}1 \text { day/ } \\
\text { month }\end{array}$ & $6(15 \%)$ & $6(15 \%)$ & $7(17.5 \%)$ & $\begin{array}{l}5 \\
(12.5 \%)\end{array}$ & $6(15 \%)$ & $6(15 \%)$ \\
\hline & $\begin{array}{l}2- \\
3 / \text { month }\end{array}$ & $2(5 \%)$ & $2(5 \%)$ & $5(12.5 \%)$ & $\begin{array}{l}7 \\
(17.5 \%)\end{array}$ & $8(20 \%)$ & $\begin{array}{l}5 \\
(12.5 \%)\end{array}$ \\
\hline & $\begin{array}{l}1 \\
\text { day/week }\end{array}$ & $1(2.5 \%)$ & $4(10 \%)$ & $1(2.5 \%)$ & $\begin{array}{l}1 \\
(2.5 \%)\end{array}$ & $1(2.5 \%)$ & $4(10 \%)$ \\
\hline & $\begin{array}{l}>1 \\
\text { day/week }\end{array}$ & $6(15 \%)$ & $4(10 \%)$ & $0(0 \%)$ & $2(5 \%)$ & $0(0 \%)$ & $2(5 \%)$ \\
\hline & Daily & 5 (12.5\%) & $\begin{array}{l}3 \\
(7.5 \%)\end{array}$ & $0(0 \%)$ & $0(0 \%)$ & $0(0 \%)$ & $0(0 \%)$ \\
\hline \multirow[t]{4}{*}{ Vomiting } & $\begin{array}{l}<1 \\
\text { day/month }\end{array}$ & $24(60 \%)$ & $\begin{array}{l}24 \\
(60 \%)\end{array}$ & 37 (92.5\%) & $\begin{array}{l}29 \\
(72.5 \%)\end{array}$ & 35 (87.5\%) & $\begin{array}{l}27 \\
(67.5 \%)\end{array}$ \\
\hline & $\begin{array}{l}1 \text { day/ } \\
\text { month }\end{array}$ & $8(20 \%)$ & $\begin{array}{l}11 \\
(27.5 \%)\end{array}$ & $3(7.5 \%)$ & $\begin{array}{l}7 \\
(17.5 \%)\end{array}$ & $4(10 \%)$ & $\begin{array}{l}9 \\
(22.5 \%)\end{array}$ \\
\hline & $\begin{array}{l}2- \\
3 / \text { month }\end{array}$ & $3(7.5 \%)$ & $\begin{array}{l}3 \\
(7.5 \%)\end{array}$ & $0(0 \%)$ & $\begin{array}{l}3 \\
(7.5 \%)\end{array}$ & $1(2.5 \%)$ & $2(5 \%)$ \\
\hline & $\begin{array}{l}1 \\
\text { day/week }\end{array}$ & $2(5 \%)$ & $0(0 \%)$ & $0(0 \%)$ & $\begin{array}{l}1 \\
(2.5 \%)\end{array}$ & $0(0 \%)$ & $\begin{array}{l}1 \\
(2.5 \%)\end{array}$ \\
\hline
\end{tabular}




\begin{tabular}{|c|c|c|c|c|c|c|c|}
\hline \multirow[t]{2}{*}{ Variables } & \multirow[t]{2}{*}{ Frequency } & \multicolumn{2}{|l|}{ Baseline } & \multicolumn{2}{|l|}{ After 4 weeks } & \multicolumn{2}{|c|}{ After 8 weeks } \\
\hline & & Intervention & Control & Intervention & Control & Intervention & Control \\
\hline & $\begin{array}{l}>1 \\
\text { day/week }\end{array}$ & $1(2.5 \%)$ & $2(5 \%)$ & $0(0 \%)$ & $0(0 \%)$ & $0(0 \%)$ & $\begin{array}{l}1 \\
(2.5 \%)\end{array}$ \\
\hline & Daily & $2(5 \%)$ & $0(0 \%)$ & $0(0 \%)$ & $0(0 \%)$ & $0(0 \%)$ & $0(0 \%)$ \\
\hline & & 0.440 & & 0.087 & & 0.259 & \\
\hline \multirow[t]{7}{*}{$\begin{array}{l}\text { Epigastric } \\
\text { pain }\end{array}$} & $\begin{array}{l}<1 \\
\text { day/month }\end{array}$ & $2(5 \%)$ & $\begin{array}{l}3 \\
(7.5 \%)\end{array}$ & 13 (32.5\%) & $\begin{array}{l}5 \\
(12.5 \%)\end{array}$ & $8(20 \%)$ & $\begin{array}{l}5 \\
(12.5 \%)\end{array}$ \\
\hline & $\begin{array}{l}1 \text { day/ } \\
\text { month }\end{array}$ & $2(5 \%)$ & $\begin{array}{l}3 \\
(7.5 \%)\end{array}$ & $14(35 \%)$ & $\begin{array}{l}7 \\
(17.5 \%)\end{array}$ & 15 (37.5\%) & $\begin{array}{l}7 \\
(7.5 \%)\end{array}$ \\
\hline & $\begin{array}{l}2- \\
3 / \text { month }\end{array}$ & $1(2.5 \%)$ & $6(15 \%)$ & $13(32.5 \%)$ & $\begin{array}{l}9 \\
(22.5 \%)\end{array}$ & 15 (37.5\%) & $8(20 \%)$ \\
\hline & $\begin{array}{l}1 \\
\text { day/week }\end{array}$ & $6(15 \%)$ & $8(20 \%)$ & $0(0 \%)$ & $\begin{array}{l}11 \\
(27.5 \%)\end{array}$ & $2(5 \%)$ & $\begin{array}{l}11 \\
(27.5 \%)\end{array}$ \\
\hline & $\begin{array}{l}>1 \\
\text { day/week }\end{array}$ & $14(35 \%)$ & $\begin{array}{l}11 \\
(27.5 \%)\end{array}$ & $0(0 \%)$ & $6(15 \%)$ & $0(0 \%)$ & $6(15 \%)$ \\
\hline & Daily & 15 (37.5\%) & $\begin{array}{l}9 \\
(22.5 \%)\end{array}$ & $0(0 \%)$ & $2(5 \%)$ & $0(0 \%)$ & $\begin{array}{l}3 \\
(7.5 \%)\end{array}$ \\
\hline & & 0.295 & & $<0.001$ & & $<0.001$ & \\
\hline \multirow[t]{7}{*}{$\begin{array}{l}\text { Epigastric } \\
\text { burning }\end{array}$} & $\begin{array}{l}<1 \\
\text { day/month }\end{array}$ & $5(12.5 \%)$ & $2(5 \%)$ & $17(42.5 \%)$ & $\begin{array}{l}5 \\
(12.5 \%)\end{array}$ & $12(30 \%)$ & $4(10 \%)$ \\
\hline & $\begin{array}{l}1 \text { day/ } \\
\text { month }\end{array}$ & $2(5 \%)$ & $\begin{array}{l}3 \\
(7.5 \%)\end{array}$ & $10(25 \%)$ & $\begin{array}{l}7 \\
(17.5 \%)\end{array}$ & 15 (37.5\%) & $6(15 \%)$ \\
\hline & $\begin{array}{l}2- \\
3 / \text { month }\end{array}$ & $4(10 \%)$ & $\begin{array}{l}7 \\
(17.5 \%)\end{array}$ & $10(25 \%)$ & $\begin{array}{l}9 \\
(22.5 \%)\end{array}$ & $10(25 \%)$ & $\begin{array}{l}7 \\
(17.5 \%)\end{array}$ \\
\hline & $\begin{array}{l}1 \\
\text { day/week }\end{array}$ & 5 (12.5\%) & $\begin{array}{l}5 \\
(12.5 \%)\end{array}$ & $3(7.5 \%)$ & $\begin{array}{l}9 \\
(22.5 \%)\end{array}$ & $3(7.5 \%)$ & $\begin{array}{l}12 \\
(30 \%)\end{array}$ \\
\hline & $\begin{array}{l}>1 \\
\text { day/week }\end{array}$ & $14(35 \%)$ & $\begin{array}{l}14 \\
(35 \%)\end{array}$ & $0(0 \%)$ & $\begin{array}{l}9 \\
(22.5 \%)\end{array}$ & $0(0 \%)$ & $\begin{array}{l}10 \\
(25 \%)\end{array}$ \\
\hline & Daily & $10(25 \%)$ & $\begin{array}{l}9 \\
(22.5 \%)\end{array}$ & $0(0 \%)$ & $\begin{array}{l}1 \\
(2.5 \%)\end{array}$ & $0(0 \%)$ & $\begin{array}{l}1 \\
(2.5 \%)\end{array}$ \\
\hline & & 0.798 & & $<0.001$ & & $<0.001$ & \\
\hline
\end{tabular}

\subsection{Drug adverse effects}

The patients didn't report any serious adverse effects. Mild bloating, and mild diarrhea were reported by three patients in the placebo group. Two patients in the herbal formula group reported mild to moderate diarrhea. No significant difference was observed between the two groups. 


\section{Discussion And Conclusion 4.1. Overall evidence}

This present study indicated the significant efficacy of the herbal formula of $B$. persicum, and $C$. sativum in the improvement of symptom severity, symptom frequency, and the patient's quality of life. Although this herbal formula can ameliorate both epigastric pain syndrome (EPS) and postprandial distress syndrome (PDS); bloating, postprandial fullness, epigastric pain, early satiety, and belching appear to have improved higher than epigastric burning, nausea, and vomiting.

Several studies have reported the efficacy of herbal medicine in FD. Yangwatana et al. reported a similar effect of Curcuma longa compared to Omeprazole in improving dyspepsia symptoms and the quality of life of FD patients (28). Mu et al. confirmed the effectiveness of Gingiber Officinalis on the acceleration of gastric emptying and antrum contraction in FD patients compared to the placebo (29). In a study conducted by Azimi et al., the herbal formula of Apium graveolence, and Trachyspermum copticum was found as effective as Omeprazole on symptoms and the quality of life of patients with EPS; and more effective than Omeprazole in symptoms and the quality of life of patients with PDS $(30,31)$. Khonche et al. illustrated the significant efficacy of hydroalcoholic extract of Mentha pulegium leaf in ameliorating PDS symptoms (32). Moreover, Hashem Dabbaghian et al. reported the significant efficacy of honey-based formulation of Nigella sativa in the improvement of PDS symptoms (33).

A review of literature shows that simple herbal medicines are more effective in the improvement of some FD symptoms. It seems many herbal medicines can improve PDS symptoms more than EPS symptoms. However, some compound herbal formulas such as A. graveolence and T. copticom can alleviate both PDS and EPS symptoms. In our study, despite the efficacy of herbal formula in both PDS and EPS; PDS symptoms were improved more significantly than EPS symptoms.

Although the therapeutic mechanisms of the herbal formula of B. persicum and $C$. sativum are not clearly understood, it can improve dyspepsia symptoms by ameliorating gastrointestinal mucosal inflammation, relieving gastrointestinal spasms, and stimulating gastrointestinal motility.

\section{2. Limitations Of The Study}

This study was conducted using a small sample size in the patients with functional dyspepsia. Hence, our findings cannot be generalized to all dyspeptic patients. Further studies with larger sample sizes are required for a more accurate assessment of this herbal formula.

\section{Abbreviations}

FD

Functional dyspepsia

EPS

Epigastric pain syndrome 
PDS

Postprandial distress syndrome

IRCT

Iranian Registry of Clinical Trials

CAM

Complementary and alternative medicine

FGID

Functional gastrointestinal disorders

HMG-CoA

$\beta$-Hydroxy $\beta$-methylglutaryl coenzyme $A$

QOL

Quality of life

GAE

Gallic acid equivalents

ANOVA

Analysis of covariance

MANOVA

Multivariate analysis of covariance

SPSS

Statistical Package for the Social Sciences

\section{Declarations}

\section{Ethics approval and consent to participate}

The study was conducted in accordance with the declaration of Helsinki and subsequent revisions and approved by the ethical committee of Kerman University of Medical Sciences (code: IR.KMU.REC.1398.470). All participants were assured of the confidentiality and anonymity of the study. Written informed consents were obtained before entering into the study. This study was registered in Iranian Registry of Clinical Trials (Number 20190304042911N1 on 03/11/2019).

\section{Consent for publication}

Not applicable

\section{Availability of data and materials}

Not applicable

\section{Competing interests}

All authors declare no conflict of interest related to this work.

\section{Funding}


This research received no grants from any funding agencies in the public, commercial, or not-for-profit sectors.

\section{Authors' contributions}

M. Esfandiarpour contributed in data collection, screening, and follow-up with subjects. M. Raeiszadeh contributed in drug preparation, and data analysis. A. Saidpour Parizi contributed in data collection, screening, and data management. M. Azimi contributed in all study related issues. All authors contributed in writing the manuscript.

\section{Acknowledgements}

We would like to express our gratitude to the authorities at the Kerman University of Medical Sciences for supporting this study.

\section{References}

[1] Douglas A Drossman LC, William D. Chey, John E Kellow. Functional gastrointestinal disorders, disorders of Gut-Brain interaction. edition f, editor. North Carolina: ROME Foundation, Ralegh; 2017;2: 351-373. https://doi.org/10.1053/j.gastro.2017.11.279

[2] Kim BJ, Kuo B. Gastroparesis and functional dyspepsia: a blurring distinction of pathophysiology and treatment. Journal of neurogastroenterology and motility. 2019;25(1):27. https://doi.org/10.5056/jnm18162

[3] Melashchenko S, Chernobrovyi V, Ksenchyn O. Prevalence of acidic and non-acidic refluxes in patients with functional dyspepsia and its risk factors investigated by means of multichannel intraluminal pH-monitoring. Wiadomości Lekarskie. 2019;72(4):604-12. https://doi.org/10.36740/wlek201904119

[4] Alam L, Naqvi M, Saeed F. Relation of Functional Dyspepsia with anxiety and depression and its impact on the quality of life of patients. PAFMJ. 2019;69(2):230-35.

[5] Madisch A, Andresen V, Enck P, Labenz J, Frieling T, Schemann M. The diagnosis and treatment of functional dyspepsia. Deutsches Ärzteblatt International. 2018;115(13):222.

https://doi.org/10.3238/arztebl.2018.0222

[6] Tomita T, Oshima T, Miwa H. New approaches to diagnosis and treatment of functional dyspepsia. Current gastroenterology reports. 2018;20(12):1-8. https://doi.org/10.1007/s11894-018-0663-4

[7] Masuy I, Van Oudenhove L, Tack J. treatment options for functional dyspepsia. Alimentary pharmacology \& therapeutics. 2019;49(9):1134-72. https://doi.org/10.1111/apt.15191

[8] Chiarioni G, Pesce M, Fantin A, Sarnelli G. Complementary and alternative treatment in functional dyspepsia. United European gastroenterology journal. 2018;6(1):5-12.

https://doi.org/10.1177/2050640617724061

[9] Wang, Y., Liu, B., Fu, X., Tong, T., Yu, Z. Efficacy and safety of Si-Jun-Zi-Tang-based therapies for functional (non-ulcer) dyspepsia: a meta-analysis of randomized controlled trials. BMC Complementary Medicine and 
Therapies, 2021; 21(1), 1-11. https://doi.org/10.1186/s12906-020-03176-z

[10] Majidi Z, Bina F, Kahkeshani N, Rahimi R. Bunium persicum: a review of ethnopharmacology, phytochemistry, and biological activities. Traditional and Integrative Medicine. 2020.

https://doi.org/10.18502/tim.v5i3.4322

[11] Bansal S, Sharma K, Gautam V, Lone AA, Malhotra EV, Kumar S, et al. A Comprehensive Review of Bunium persicum: A Valuable Medicinal Spice. Food Reviews International. 2021:1-20.

https://doi.org/10.1080/87559129.2021.1929305

[12] Yousefi SS, Sadeghpour O, Hamzehgardeshi Z, Sohrabvand F. The effects of Carum carvi (Bunium persicum Boiss) on early return of bowel motility after Caesarean section: double-blind, randomized, placebocontrolled trial. Journal of family \& reproductive health. 2019;13(1):35.

https://doi.org/10.18502/jfrh.v13i1.1611

[13] Kazemipoor M, Radzi CWJBWM, Hajifaraji M, Cordell GA. Preliminary Safety Evaluation and Biochemical Efficacy of a Carum carvi Extract: Results from a Randomized, Triple-Blind, and Placebo-Controlled Clinical Trial. Phytotherapy research. 2014;28(10):1456-60. https://doi.org/10.1002/ptr.5147

[14] Keshavarz A, Minaiyan M, Ghannadi A, Mahzouni P. Effects of Carum carvi L.(Caraway) extract and essential oil on TNBS-induced colitis in rats. Research in pharmaceutical sciences. 2013;8(1):1.

[15]Khajeali Y, Kheiri F, Rahimian Y, Faghani M, Amjo AN. Effect of use different levels of caraway (Carum carvi L.) powder on performance, some blood parameters and intestinal morphology on broiler chicks. World Applied Sciences Journal. 2013;24(8):1044-8.

[16] Al-Essa MK, Shafagoj YA, Mohammed Fl, Afifi FU. Relaxant effect of ethanol extract of Carum carvi on dispersed intestinal smooth muscle cells of the guinea pig. Pharmaceutical biology. 2010;48(1):76-80. https://doi.org/10.3109/13880200903046161

[17] Prachayasittikul V, Prachayasittikul S, Ruchirawat S, Prachayasittikul V. Coriander (Coriandrum sativum): A promising functional food toward the well-being. Food Research International. 2018;105:305-23. https://doi.org/10.1016/j.foodres.2017.11.019

[18] Wei J-N, Liu Z-H, Zhao Y-P, Zhao L-L, Xue T-K, Lan Q-K. Phytochemical and bioactive profile of Coriandrum sativum L. Food chemistry. 2019;286:260-7. https://doi.org/10.1016/j.foodchem.2019.01.171

[19] Sabetan Shiraze AA, Agah MJ, Hasanabadi A, Boostani A. Effect of dietary Coriander seed (Coriandrum sativum L.) and a-tocopheryle acetate supplementation on growth performance, intestinal morphology and nutrient digestibility in broiler chickens. Animal Sciences Journal. 2018;31(120):205-16.

[20] Bijukumar BS. A Study on the Effect of Dietary Fibre on Gastro-Intestinal Motility in Rats. Journal of Advances in Biological Science. 2018;5(2):76-78

[21] Al-Mofleh IA, Alhaider AA, Mossa JS, Al-Sohaibani MO, Rafatullah S, Qureshi S. Protection of gastric mucosal damage by Coriandrum sativum L. pretreatment in Wistar albino rats. Environmental Toxicology and 
Pharmacology. 2006;22(1):64-9. https://doi.org/10.1016/j.etap.2005.12.002

[22] Jahan N, Nikhat S, Ahmad G. Efficacy of Tukhm Kishneez (Coriandrum sativum Linn.) in stress induced gastric ulcer. Unani Research. 2011;1(1):17-22.

[23] Raeiszadeh M, Pardakhty A, Sharififar F, Mehrabani M. Phytoniosome: a novel drug delivery for myrtle extract. Iranian journal of pharmaceutical research: IJPR. 2018;17(3):804. https://doi.org/10.4103/17355362.228955

[24] Linda Rabeneck, Karon F Cook, Kimberly Wristers, Julianne Souchek, Terri Menke, Nelda P Wray. SODA (severity of dyspepsia assessment): A new effective outcome measure for dyspepsia-related health. Journal of Clinical Epidemiology. 2001;54(8): 755-765. https://doi.org/10.1016/s0895-4356(00)00365-6

[25] Palsson OS, Whitehead WE, Van Tilburg MAL, Chang L, Chey W, Crowell MD, et al. Development and validation of the Rome IV diagnostic questionnaire for adults. Gastroenterology. 2016;150(6):1481-91. https://doi.org/10.1053/j.gastro.2016.02.014

[26] Talley NJ. Quality of life in functional dyspepsia: responsiveness of the Nepean Dyspepsia Index and development of a new 10-item short form. Alimentary Pharmacology \& Therapeutics. 2001;15(2):20716. https://doi.org/10.1046/j.1365-2036.2001.00900.x

[27] Maryam Azimi, Farzaneh Zolala, Mohammad Reza Baneshi, Mohammad Javad Zahedi. Validation of Short-form Nepean Dyspepsia Index (NDI-10) in Iranian patients with Functional Dyspepsia (FD). GOVARESH. 2017;22(2):17-27.

[28]Yongwatana K, Harinwan K, Chirapongsathorn S, Opuchar K, Sanpajit T, Piyanirun W, et al. 813-Curcuma Longa Linn Versus Omeprazole in Treatment of Functional Dyspepsia, a Randomized, Double-Blind, PlaceboControlled Trial. Gastroenterology. 2019;156(6):S-171 https://doi.org/10.1016/s0016-5085(19)37219-1

[29]Hu ML, Rayner CK, Wu KL, Chuah SK, Tai WC, Chou YP, et al. Effect of ginger on gastric motility and symptoms of functional dyspepsia. World J Gastroenterol. 2011;17(1):105-

10. https://doi.org/10.3748/wjg.v17.i1.105

[30] Azimi M, Zahedi MJ, Mehrabani M, Tajadini H, Zolala F, Baneshi MR, et al. Effect of Apium graveolens and Trachyspermum copticom on clinical symptoms of patients with functional dyspepsia. Avicenna Journal of Phytomedicine. 2017;7(6):554-64.

[31] Azimi M, Zahedi MJ, Mehrabani M, Tajadini H, Zolala F, Baneshi MR, et al. Effects of Iranian Traditional Medicine Remedies (Apium Graveolence and Trachyspermum Copticum) on Modifying the Quality of Life in Patients with Functional Dyspepsia: A Double-Blind Randomized Clinical Trial. Galen Med J. 2017;6(2):102-9.

[32] Khonche A, Fallah Huseini H, Abdi H, Mohtashami R, Nabati F, Kianbakht S. Efficacy of Mentha pulegium extract in the treatment of functional dyspepsia: A randomized double-blind placebo-controlled clinical trial. J Ethnopharmacol. 2017;206:267-73. https://doi.org/10.1016/j.jep.2017.05.026 
[33] Hashem-Dabaghian F, Agah S, Taghavi-Shirazi M, Ghobadi A. Combination of Nigella sativa and Honey in Eradication of Gastric Helicobacter pylori Infection. Iran Red Crescent Med J.

2016;18(11):e23771. https://doi.org/10.5812/ircmj.23771

\section{Figures}

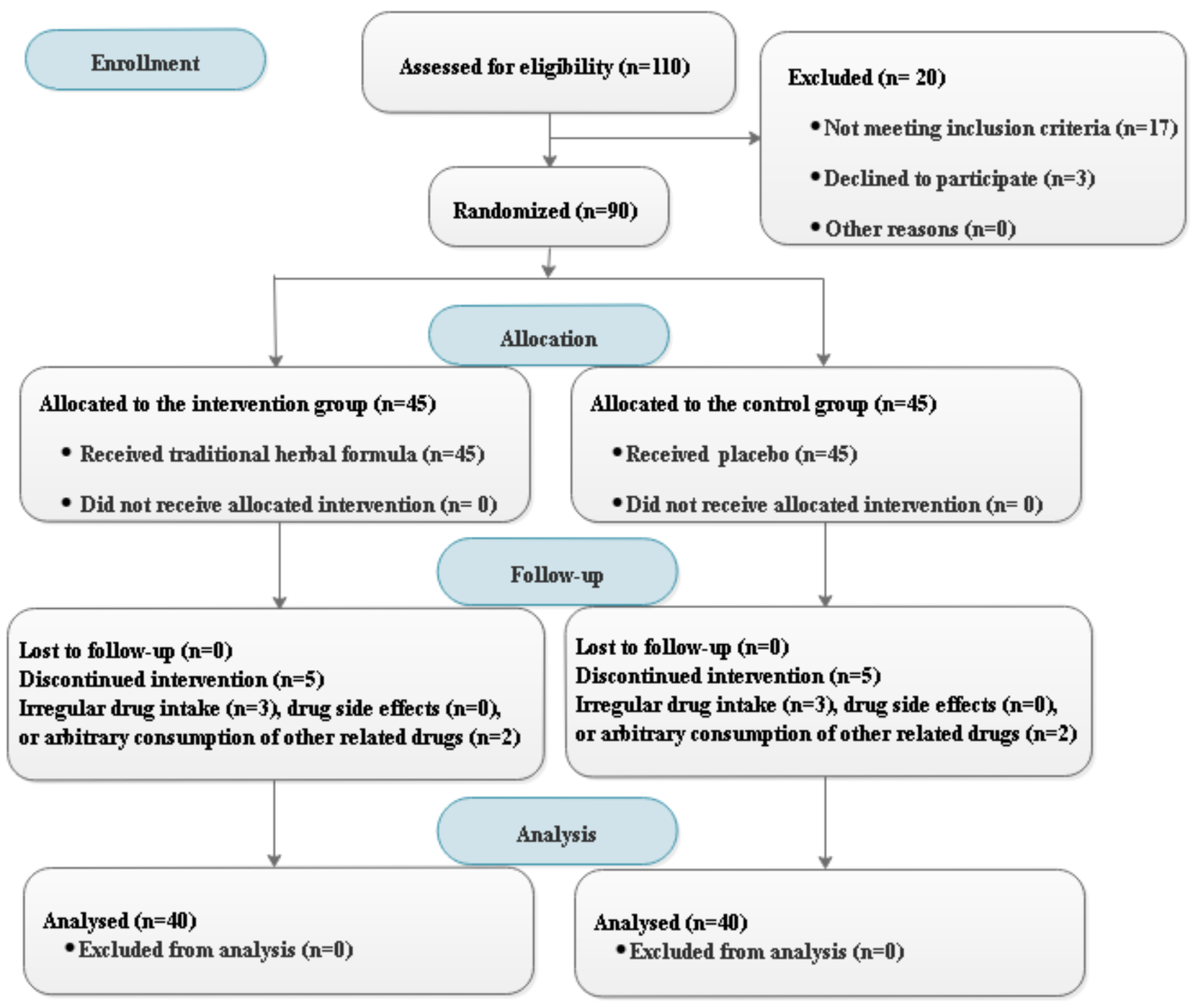

Figure 1

Flowchart of the study

\section{Supplementary Files}

This is a list of supplementary files associated with this preprint. Click to download.

- CONSORT2010ChecklistA3.doc 